\title{
Knockdown of metadherin inhibits cell proliferation and migration in colorectal cancer
}

\author{
JIAN-WANG LI ${ }^{1 *}$, CHUN-ZHEN HUANG $^{1 *}$, JIAN-HUA LI $^{3}$, JIAN-HUA YUAN $^{1}$, QIONG-HUI CHEN ${ }^{1}$, \\ WEI-FANG ZHANG ${ }^{1}$, ZHEN-SHENG XU ${ }^{1}$, YING-PING LIU ${ }^{1}$, YONG LI $^{2}$, \\ MEI-XIAO ZHAN ${ }^{2}$ and LI-GONG LU ${ }^{2}$
}

\author{
${ }^{1}$ Department of Oncology, Central South University Xiangya School of Medicine Affiliated Haikou Hospital/ \\ Haikou People's Hospital, Haikou, Hainan 570208; ${ }^{2}$ Interventional Radiology Center, Zhuhai Precision Medicine Center, \\ Zhuhai People's Hospital, Zhuhai Hospital Affiliated with Jinan University, Zhuhai, Guangdong 519000; \\ ${ }^{3}$ Department of General Surgery, Fuzhou People's Hospital, Fuzhou, Jiangxi 344000, P.R. China
}

Received October 26, 2017; Accepted July 12, 2018

DOI: $10.3892 / o r .2018 .6581$

\begin{abstract}
Metadherin (MTDH) is a multifunctional oncogene involved in tumor cell migration and metastasis through regulating a number of oncogenic signaling pathways in various human malignancies. Previous studies have demonstrated that MTDH is overexpressed in human colorectal cancer (CRC) and associated with cancer progression and a poor prognosis. However, the underlying mechanisms remain largely unknown. The present study investigated the expression and role of MTDH in CRC cells as well as the underlying mechanism of this. Western blot analysis and quantitative polymerase chain reaction were conducted to determine protein and mRNA expression of MTDH in three human CRC cell lines. A short hairpin RNA (shRNA) targeting MTDH was introduced into CRC HCT116 cells to stably inhibit MTDH expression. A Cell Counting Kit- 8 assay, colony formation assay, Transwell assay and flow cytometry were used to investigate the effect of MTDH-knockdown on cell proliferation, migration, apoptosis and cell cycle arrest. Western blotting was performed to examine the protein expression levels of cell growth- and apoptosis-associated genes. The results demonstrated that MTDH was commonly expressed in CRC cell lines. MTDH silencing significantly suppressed cell growth, colony forming ability and migration while inducing the apoptosis of HCT116 cells. In addition, MTDH depletion induced S phase cell cycle arrest in HCT116 cells. Mechanistically, knockdown of MTDH markedly downregulated the expression of phosphorylated
\end{abstract}

Correspondence to: Li-Gong Lu, Interventional Radiology Center, Zhuhai Precision Medicine Center, Zhuhai People's Hospital, 79 Kangning Road, Zhuhai, Guangdong 519000, P.R. China

E-mail: luligong1969@126.com

*Contributed equally

Key words: colorectal cancer, metadherin, proliferation, protein kinase B/c-Myc pathway protein kinase $\mathrm{B}, \mathrm{c}-\mathrm{Myc}$, proliferating cell nuclear antigen and B-cell lymphoma 2 (Bcl-2) protein in HCT116 cells, and the expression of $\mathrm{p} 53$ and $\mathrm{Bcl}-2$-associated $\mathrm{X}$ protein was significantly increased compared with the negative control shRNA group $(\mathrm{P}<0.05)$, suggesting that MTDH may function through the expression of numerous types of apoptosis-associated and signaling channel proteins in CRC cells. Taken together, these data indicated that MTDH may serve as a biomarker and candidate therapeutic target for CRC.

\section{Introduction}

Colorectal cancer (CRC) is the third most common type of cancer worldwide, accounting for $8 \%$ of all cancer-related mortality (1). There is an urgent requirement to identify potential therapeutic targets to effectively inhibit CRC cell growth, invasion and metastasis $(2,3)$. It is well-established that cancer progression is associated with genetic and epigenetic alterations along with the constructional changes in the CRC microenvironment. The activities of several signaling pathways, including Ras, Wnt and Myc signaling, have been correlated with CRC carcinogenesis, which provides potential biomarkers for early diagnosis and prognosis of CRC as well as therapeutic targets for CRC $(4,5)$.

Metadherin (MTDH), also known as astrocyte elevated gene 1 (AEG-1) and lysine-rich CEACAM1 co-isolated (LYRIC), is located on chromosome 8q22 and encodes a single-pass transmembrane protein (6). MTDH has been reported to be overexpressed in solid tumors and promotes tumor cell proliferation, migration and invasion (7-10). Clinical studies have also demonstrated that MTDH overexpression is associated with tumorigenesis, tumor development and short survival times in hepatocellular carcinoma (HCC), gastric and breast cancer, and CRC (11-14). MTDH-mediated tumor progression is regulated by multiple signaling pathways, including nuclear factor- $\kappa \mathrm{B}(\mathrm{NF}-\kappa \mathrm{B})$, phosphoinositide 3-kinase (PI3K)/protein kinase B (Akt), mitogen-activated protein kinase (MAPK)/extracellular signal-regulated kinase (ERK) and Wnt (15-18). The overexpression of MTDH in CRC is significantly correlated with 
the Union for International Cancer Control stages, Ki-67 expression, histological differentiation and shorter survival times (19). However, the expression and role of MTDH in CRC as well as the underlying mechanism remain largely unknown.

The present study determined the protein and mRNA expression level of MTDH in human CRC cell lines and investigated its role in CRC cell behavior, including proliferation, colony-forming and migratory abilities, cell cycle arrest, and apoptosis in vitro. The present study also investigated the underlying molecular mechanism of MTDH-regulated CRC growth by detecting the protein expression of important cell growth- and apoptosis-associated genes in MTDH-deficient CRC cells.

\section{Materials and methods}

Cell lines. CRC SW480, HCT116 and LoVo cell lines (Type Culture Collection of the Chinese Academy of Sciences, Shanghai, China), and the normal colonic mucosa epithelial NCM460 cell line (American Type Culture Collection, Manassas, VA, USA) were cultured in Dulbecco's modified Eagle's medium (DMEM; Corning Incorporated, Corning, NY, USA), containing $10 \%$ fetal bovine serum (FBS; Shanghai VIAN-SAGA Biotech Ltd., Shanghai, China), streptomycin $(100 \mu \mathrm{g} / \mathrm{ml})$, and penicillin $(100 \mathrm{IU} / \mathrm{ml})$ at $37^{\circ} \mathrm{C}$ in a humidified atmosphere of $5 \% \mathrm{CO}_{2}$.

Reverse transcription polymerase chain reaction (RT-PCR). Extraction of total RNA from cells was performed using TRIzol reagent (Invitrogen; Thermo Fisher Scientific, Inc. Waltham, MA, USA). RNA was reverse transcribed to cDNA using a PrimeScript RT reagent kit (Takara Biotechnology Co., Ltd., Dalian, China). PCR amplification was conducted using the following primer sequences: MTDH forward, 5'-AAGCAG TGCAAAACAGTTCACG-3' and reverse, 5'-GCACCTTAT CACGTTTACGCT-3'; and GAPDH forward, 5'-TGACTT CAACAGCGACACCCA-3' and reverse, 5'-CACCCTGTT GCTGTAGCCAAA-3'. The thermocycling conditions were $95^{\circ} \mathrm{C}$ for $15 \mathrm{sec}, 45 \mathrm{cycles}$ at $95^{\circ} \mathrm{C}$ for $5 \mathrm{sec}$, and $60^{\circ} \mathrm{C}$ for $30 \mathrm{sec}$.

Lentivirus-mediated short hairpin RNA (Lenti-shRNA) against $M T D H$. The Lenti-shRNA vector system (pGCSIL-GFP-puromycin) was constructed, packaged and purified by Shanghai GeneChem Co., Ltd. (Shanghai, China). Cells were seeded in 6-well plates at $3 \times 10^{5}$ cells/well using Lipofectamine 2000 transfection reagent (Invitrogen; Thermo Fisher Scientific, Inc.) according to the manufacturer's protocol. The following 3 specific MTDH short hairpin RNAs (shRNAs): shMTDH-1, 5'AGGAATAAAGGATTCTGAT3'; shMTDH-2, 5'-AAG TCAAATACCAAGCAAA-3'; and shMTDH-3, 5'-AACTTA CAACCGCATCATT-3', as well as a negative control shRNA (shNC), 5'-TTCTCCGAACGTGTCACGT-3'. The cells were cultured for the next $48 \mathrm{~h}$ and were then harvested for RT-PCR and simple western blot analysis or prepared for the following experiments.

RT-quantitative PCR (RT-qPCR). Isolation of total RNA was conducted using TRIzol reagent (Invitrogen; Thermo Fisher Scientific, Inc.). The sequences of the primers (Shanghai GeneChem Co., Ltd.) used for qPCR were as follows: MTDH forward, 5'-AAGCAGTGCAAAACAGTTCACG-3' and reverse, 5'-CACCCTGTTGCTGTAGCCAAA-3'; and GAPDH forward, 5'-AAGCAGTGCAAAACAGTTCACG-3' and reverse, 5'-GCACCTTATCACGTTTACGCT-3'. qPCR was performed using a SYBR Master Mix (Takara Biotechnology Co., Ltd.) on a Stratagene MX3000p Real-time PCR thermocycler (Agilent Technologies, Inc., Santa Clara, CA, USA) with an initial denaturation step at $95^{\circ} \mathrm{C}$ for $10 \mathrm{~min}$, followed by 45 cycles of $95^{\circ} \mathrm{C}$ for $3 \mathrm{sec}$ and $60^{\circ} \mathrm{C}$ for $30 \mathrm{sec}$. The experiments were performed in triplicate. mRNA levels of MTDH were analyzed using the $2^{-\Delta \Delta \mathrm{Cq}}$ method (20).

Size-based simple and traditional western blot analyses. MTDH proteins following shRNA-mediated knockdown for simple western analysis of total receptor levels were diluted to a final protein concentration of $1 \mathrm{X}$ sample buffer, $1 \mathrm{X}$ fluorescent molecular weight markers and $40 \mathrm{mM}$ DTT, prior to being heated at $95^{\circ} \mathrm{C}$ for $5 \mathrm{~min}$ in a Master mix (ProteinSimple, San Jose, CA, USA) and processed at room temperature in a Sally Sue Simple Western instrument (ProteinSimple). Proteins were subsequently incubated with anti-MTDH (cat. no., Ab45338; dilution, 1:20; Abcam, Cambridge, MA, USA) and anti- $\beta$-actin (cat no., SC-69879; dilution, 1:50; Santa Cruz Biotechnology, Inc., Dallas, TX, USA) primary antibodies, Wes $12-230 \mathrm{kDa}$ Master kit with Split Buffer (cat. nos., Ab45338 and 77960; ProteinSimple) and Wes 12-230 kDa Rabbit Master Kit (cat. nos., Ab45338 and 77961; ProteinSimple). Milk-free Antibody Diluent II (cat. no., ZLI-9030; dilution, 1:50; OriGene Technologies, Inc., Beijing, China) was used to dilute primary antibodies and as a blocking reagent at $4^{\circ} \mathrm{C}$ for $2 \mathrm{~h}$. Proteins were then incubated with anti-rabbit (cat no., PS-MK14; dilution, 1:1,000; ProteinSimple) immunoglobulin G antibodies or anti-mouse immunoglobulin $\mathrm{G}$ antibodies (cat no., PS-MK15; dilution, 1:1,000; ProteinSimple) secondary antibodies. All antibodies were diluted in Immunobooster (Bioworld Technology, Inc., St. Louis Park, MN, USA) or antibody diluent (ProteinSimple) with a dilution of $1: 50$ or 1:100. All antibody incubations were performed at $4^{\circ} \mathrm{C}$ for $10-15 \mathrm{~min}$ with Immunobooster or 60-120 min with antibody diluents. Simple Western assay buffers, nano-volume capillaries and the prepared assay plate were placed in Simon (ProteinSimple), which performs all assay steps automatically. Next Luminol-S and peroxide (ProteinSimple) were added to produce chemiluminescence, which was captured by a CCD camera. Compass 2.5.11 Software (ProteinSimple) was used to analyze digital images. Of the three cell lines, the highest level of MTDH mRNA and protein expression was observed in the HCT116 cells and therefore, these cells were used to examine the function of MTDH in CRC cell behavior in the subsequent experiments.

Traditional western blot analysis was performed. Proteins were isolated using lysis buffer containing $1 \mathrm{mM}$ EDTA, $50 \mathrm{mM}$ Tris- $\mathrm{HCl}, 1 \% \mathrm{NP} 40,0.1 \%$ SDS, $150 \mathrm{mM} \mathrm{NaCl}$ and protease inhibitor. Protein concentrations were measured using a bicinchoninic acid protein assay kit. A total of $5 \mu \mathrm{g}$ protein/lane was loaded onto a $12 \%$ SDS-PAGE gel and separated, followed by transfer onto a polyvinylidene difluoride membrane. The membrane was then blocked with $2 \%$ dry skimmed milk in Tris-buffered saline with Tween-20 
(TBST) for $1 \mathrm{~h}$ at room temperature, prior to being incubated with primary antibodies against C-Myc (cat no., 6341S; dilution, 1:1,000; Cell Signaling Technology, Inc., Danvers, MA, USA), GAPDH (cat. no., SC-32233, dilution, 1:4,000; Santa Cruz Biotechnology, Inc.), B-cell lymphoma 2 (Bcl-2; dilution, 1:1,000; Abcam), Bcl-2-associated X protein (BAX; cat. no., AB7977; dilution, 1:1,000; Abcam), phosphorylated protein kinase B (p-Akt; cat. no., 13038; dilution, 1:1,000; Cell Signaling Technology, Inc.), Akt (cat no., 9272, dilution, 1:1,000; Cell Signaling Technology, Inc.), p53 (cat no., 2527, dilution, 1:1,000; Cell Signaling Technology, Inc.) and proliferating cell nuclear antigen (PCNA; cat. no., 2586S; dilution, 1:1,000; Cell Signaling Technology, Inc.) overnight at $4^{\circ} \mathrm{C}$. Following three rinses with TBST, the membrane was incubated with a horseradish peroxidase-conjugated rabbit IgG secondary antibody (cat no., sc-2004; dilution, 1:5,000; Santa Cruz Biotechnology, Inc.) and mouse IgG (cat no., sc-2005; dilution, 1:5,000; Santa Cruz Biotechnology, Inc.) for $1 \mathrm{~h}$ at room temperature. The protein bands were detected using an enhanced chemiluminescence kit (Thermo Fisher Scientific, Inc.) and analyzed using Bio-Rad 680 Quantity One software (Bio-Rad Laboratories, Inc., Hercules, CA, USA).

Cell proliferation assay. The proliferation rate of HCT116 cells was evaluated by a Cell Counting Kit-8 (CCK-8; Sigma-Aldrich; Merck KGaA, Darmstadt, Germany). A total of 5,000 stably-transfected cells were seeded into each well of a 96-well plate in 6 replicates and grown overnight. A total of $10 \mu \mathrm{l} \mathrm{CCK}-8$ reagent was added into each well at different time points. Following incubation at $37^{\circ} \mathrm{C}$ for $2 \mathrm{~h}$, absorbance was measured at $450 \mathrm{~nm}$ with a Bio-Rad plate reader (Bio-Rad Laboratories, Inc.).

Colony formation assay. A total of 2,000 stably-transfected HCT116 cells were plated into each well of a 6 -well plate in triplicate. Following incubation at $37^{\circ} \mathrm{C}$ for 10 days, the cells were stained with crystal violet for $10 \mathrm{~min}$ at room temperature. Colonies containing $>50$ cells were counted under an Olympus IX71 inverted light microscope (magnification, x100; Olympus Corporation, Tokyo, Japan). The images were captured using an Olympus digital camera (Olympus Corporation).

Cell apoptosis assay. Stably-transfected HCT116 cells were collected and resuspended in binding buffer (Invitrogen; Thermo Fisher Scientific, Inc.). On the seventh day after transfection, cell apoptosis was examined using the Annexin V-allophycocyanin (APC) apoptosis detection kit (cat. no., 88-8007-72; eBioscience; Thermo Fisher Scientific, Inc.) according to the manufacturer's protocols. A FACS Calibur flow cytometer (cat. no., 557706; BD Biosciences, Franklin Lakes, NJ, USA) was used to analyze the percentage of apoptotic cells. The cells were stained with $100 \mu \mathrm{l}$ cell suspension containing $5 \mu \mathrm{l}$ Annexin V-APC at room temperature in the dark for 10-15 min. All experiments were performed in triplicate. WinMDI 2.8 software was used for analysis.

Cell cycle analysis. Stably-transfected HCT116 cells were collected, rinsed with PBS and fixed with $70 \%$ ethanol overnight at $4^{\circ} \mathrm{C}$. Following a $30 \mathrm{~min}$ incubation with $100 \mu \mathrm{g} / \mathrm{ml}$ RNase A,
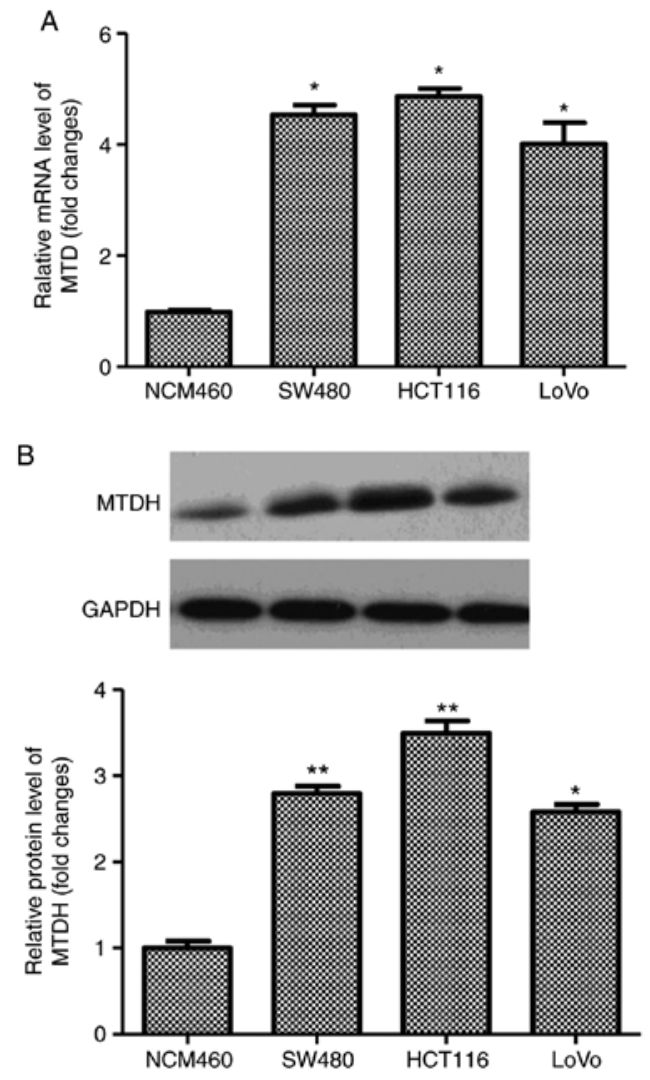

Figure 1. MTDH is highly expressed in CRC cell lines. (A) Reverse transcription-quantitative polymerase chain reaction analysis of MTDH mRNA in three human CRC cell lines (SW480, HCT116 and LoVo) and the normal colonic mucosa epithelial NCM460 cell line. (B) Western blot analysis of MTDH protein in three human CRC cell lines (SW480, HCT116 and LoVo) and the normal colonic mucosa epithelial NCM460 cell line. Data are expressed as the mean \pm standard deviation. ${ }^{*} \mathrm{P}<0.05$ vs. NCM460 group. ${ }^{* *} \mathrm{P}<0.01$ vs. shNC group. MTDH, metadherin; $\mathrm{CRC}$, colorectal cancer.

the cells were stained with $40 \mathrm{mg} / \mathrm{ml}$ PI (cat. no., P4170; Sigma-Aldrich; Merck KGaA, Darmstadt, Germany) and $100 \mu \mathrm{g} / \mathrm{ml}$ RNase A (cat. no.,EN0531; Fermentas; Thermo Fisher Scientific, Inc.) in the dark for an additional $30 \mathrm{~min}$ at room temperature. The results were analyzed using a FACSCalibur flow cytometer (cat. no., 557706; BD Biosciences). The results were analyzed using Multicycle software (version 300; Phoenix Flow System, San Diego, CA, USA).

Transwell migration assay. Transwell assays were conducted using Transwell chambers (Corning Incorporated, Corning, NY, USA) in a 24-well format. A total of $2 \times 10^{5}$ HCT116 cells in $0.1 \%$ FBS-containing serum-free RPMI-1640 medium (Gibco; Thermo Fisher Scientific, Inc.) were loaded into the upper chamber and 30\% FBS-containing medium was added into the lower chamber. Cells were incubated for $48 \mathrm{~h}$ at $37^{\circ} \mathrm{C}$. The migrating cells stained with crystal violet at $37^{\circ} \mathrm{C}$ for $1 \mathrm{~h}$ were counted in random microscopic fields under an Olympus IX71 inverted light microscope (magnification, x100; Olympus Corporation). The experiments were performed in triplicate.

Statistical analysis. All experiments were independently repeated at least three times. Data are expressed as the mean \pm standard deviation. Statistical significance was evaluated using Student's t-test or one-way analysis of variance with 

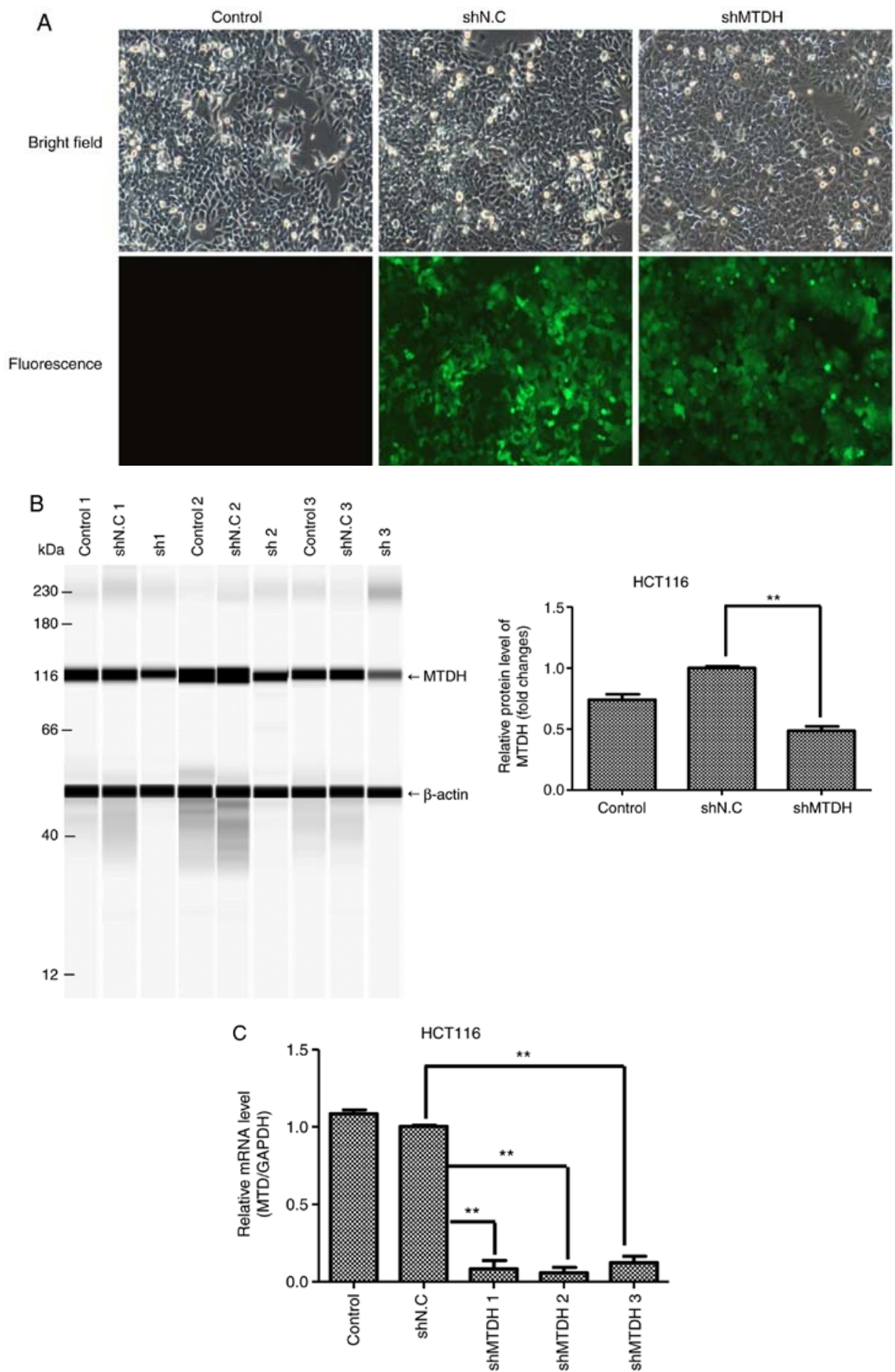

Figure 2. MTDH was effectively downregulated by the transfection of shRNA against MTDH (shMTDH) in HCT116 cells. (A) Green fluorescence under a fluorescence microscope (magnification, x100) was used to show the transfection efficiency in HCT116 cells transfected with MTDH. (B) Simple western blot analysis was conducted to detect the MTDH protein level in HCT116 cell lines following MTDH shRNA transfection. (C) Reverse transcription-quantitative polymerase chain reaction was used to measure the MTDH mRNA level in HCT116 cells following MTDH shRNA transfection. shNC, negative control shRNA; shMTDH1-3, MTDH shRNA1-3. Data are expressed as the mean \pm standard deviation. ${ }^{* *} \mathrm{P}<0.01$ vs. shNC group. MTDH, metadherin; sh/shRNA, short hairpin RNA; NC, negative control.

Prism 4.0 (GraphPad Software, Inc., La Jolla, CA, USA). P<0.05 was considered to indicate a statistically significant difference.

\section{Results}

MTDH mRNA is highly expressed in human CRC cells. The expression pattern of MTDH in human CRC SW480, HCT116 and LoVo cell lines, and normal colonic mucosa epithelial NCM460 cell line was examined by RT-qPCR and western blot analysis. The results demonstrated that the MTDH mRNA and protein expression levels were significantly higher in the human CRC cells than in the NCM460 cells (Fig. 1A and B). In addition, a higher level of MTDH mRNA was observed in HCT116 cells compared with that in the SW480 or LoVo cells. 


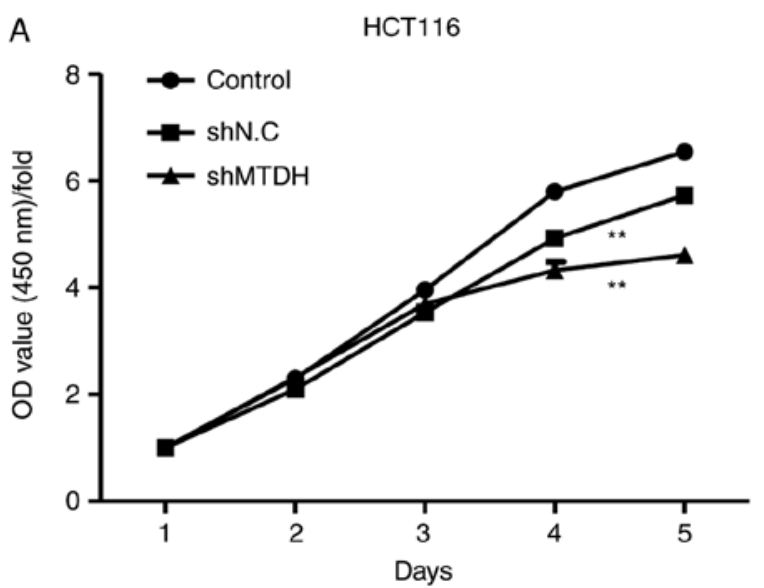

B
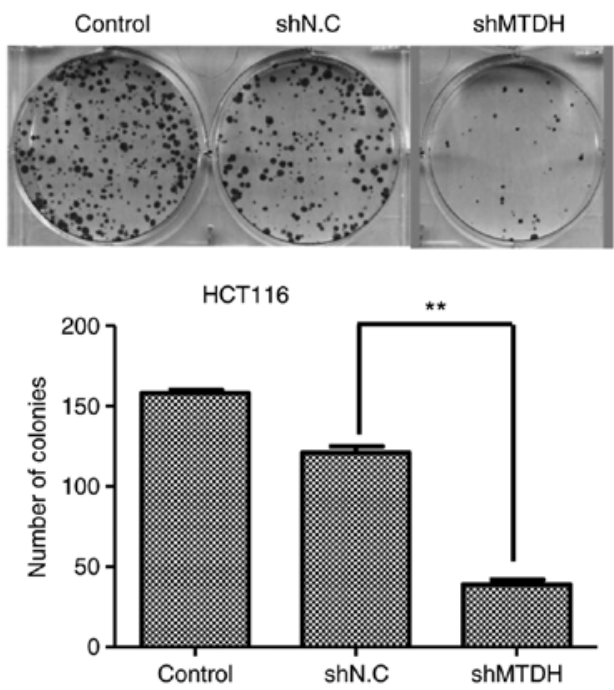

C

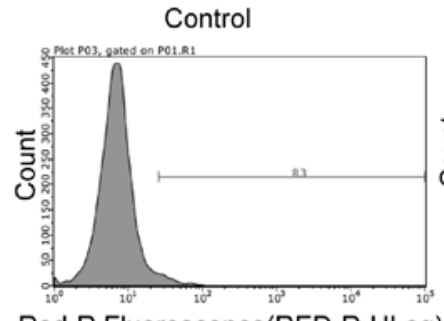

Red-R Fluorescence(RED-R-HLog) Red-R Fluorescence(RED-R-HLog) Annexin $\mathrm{V}$

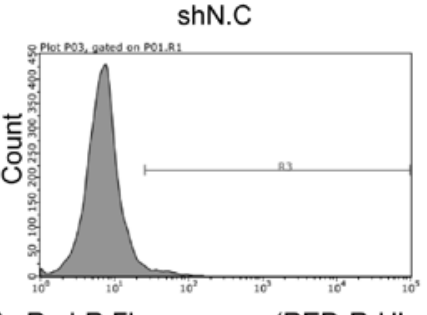

Annexin $\mathrm{V}$

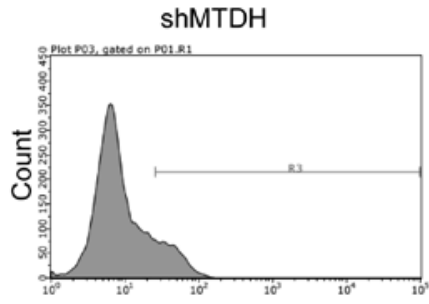

Red-R Fluorescence(RED-R-HLog)

Annexin $\mathrm{V}$

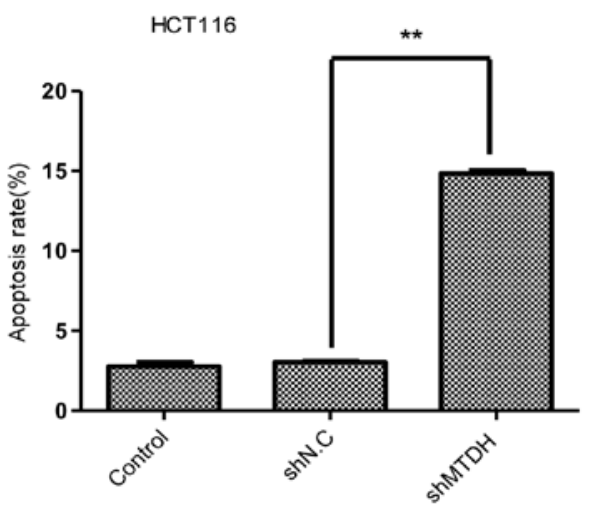

Figure 3. The effects of MTDH-knockdown on HCT116 cell proliferation and apoptosis. (A) Proliferation of HCT116 was examined using the Cell Counting Kit-8; (B) Representative images and quantification of colony formation assay; (C) Annexin V-allophycocyanin assay to assess the effects on apoptosis of MTDH-knockdown in HCT116 cells using a flow cytometer. The percentages of R3 were 2.96, 3.5 and 15.09\% in control, shNC and shMTDH groups, respectively, gated by P01.R1. Data are expressed as the mean \pm standard deviation. ${ }^{* *} \mathrm{P}<0.01$ vs. shNC group. MTDH, metadherin; sh, short hairpin RNA; NC, negative control; OD, optical density.

Therefore, HCT116 cells were used to examine the function of MTDH in CRC cell behavior in the subsequent experiments.

MTDH is essential for HCT116 cell proliferation in vitro. To investigate the role of MTDH in CRC cell proliferation, lentiviral vectors expressing shMTDH or shNC were introduced into HCT116 cells to stably silence MTDH expression. Fluorescent staining, simple western and qPCR assays were used to assess the knockdown efficacy of shMTDH. As demonstrated in Fig. 2A, cells were infected with a lentivirus containing MTDH shRNA (shMTDH) or an empty vector (shNC). The protein expression of MTDH was significantly inhibited by shMTDH in HCT116 cells compared with the negative control (Fig. 2B). Additionally, the mRNA expression of MTDH was also significantly decreased in shMTDH-transfected cells compared with that in shNC cells (Fig. 2C). These data suggested that MTDH expression was efficiently inhibited by shMTDH. The infection efficiency was $94.2 \%$ in the shMTDH-2 group relative to shNC. Since shMTDH-2 exhibited improved inhibition of MTDH expression in HCT116 cells, it was selected for subsequent experiments. To determine whether MTDH is essential for CRC cell growth, cell viability and colony formation assays were performed in a MTDH-deficient HCT116 cell line. As demonstrated in Fig. 3A, knockdown of MTDH notably inhibited 


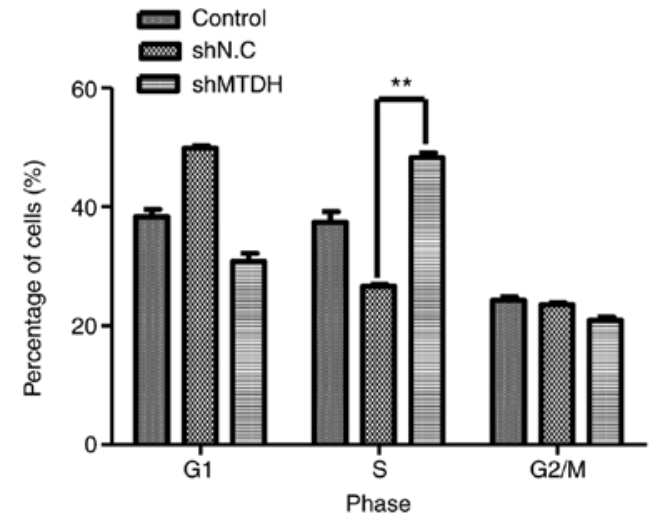

Figure 4. Knockdown of MTDH induced S phase cell cycle arrest in the HCT116 cell lines. HCT116 cells were stably transfected with shNC- or shMTDH-treated cells, or a blank control. Flow cytometry was performed for cell cycle analysis. The percentages of cells in the cell cycle phases are shown. Data are expressed as the mean \pm standard deviation. ${ }^{* *} \mathrm{P}<0.01$ vs. shNC and shMTDH groups. MTDH, metadherin; sh, short hairpin RNA; NC, negative control.

HCT116 cell proliferation compared with the negative control. In addition, a colony formation assay revealed that shMTDH markedly suppressed the colony-forming ability of HCT116 cells compared with shNC (Fig. 3B). Taken together, these data indicated that MTDH is essential for HCT116 cell growth in vitro and may serve a promotive role in CRC tumorigenesis.

Knockdown of MTDH significantly induces HCT116 cell apoptosis. Cell apoptosis is critically important in cell growth suppression (21). To investigate the mechanism underlying MTDH-mediated HCT116 cell growth, cell apoptosis analysis was performed using PI-APC-Annexin in HCT116 cells. As demonstrated in Fig. 3C, flow cytometry revealed that the percentage of apoptosis was significantly increased to $14.84 \%$ $(\mathrm{SD}=0.22 \%, \mathrm{P}<0.01)$ in the MTDH-shRNA HCT116 group, from $3.05 \%$ ( $\mathrm{SD}=0.05 \%$ ) in the shNC group, suggesting that knockdown of MTDH may induce cell apoptosis, resulting in an inhibition of cell proliferation in CRC cells.

Knockdown of MTDH induces cell cycle arrest at the Sphase. To further investigate the mechanism underlying MTDH-mediated growth and apoptosis of HCT116 cells, a cell cycle analysis was performed. As demonstrated in Fig. 4, compared with the negative control, MTDH deficiency in HCT116 cells induced a significant increase in the cell population in the S phase (48.97 vs. $26.61 \%, \mathrm{P}<0.01)$, indicating that MTDH may serve an essential role in $\mathrm{S}$ phase arrest in CRC cells.

$M T D H$ is required for CRC cell migration. To further investigate whether MTDH has an effect on CRC cell migration, a Transwell migration assay was performed to test the migratory ability of MTDH-deficient HCT116 cells. As demonstrated in Fig. 5, MTDH deficiency significantly suppressed the migratory ability of HCT116 cells compared with the negative control, suggesting that MTDH may promote CRC metastasis.

Knockdown of MTDH inhibits p-Akt and c-Myc, and increases apoptosis-related protein expression. To further investigate the underlying molecular mechanism of MTDH-mediated
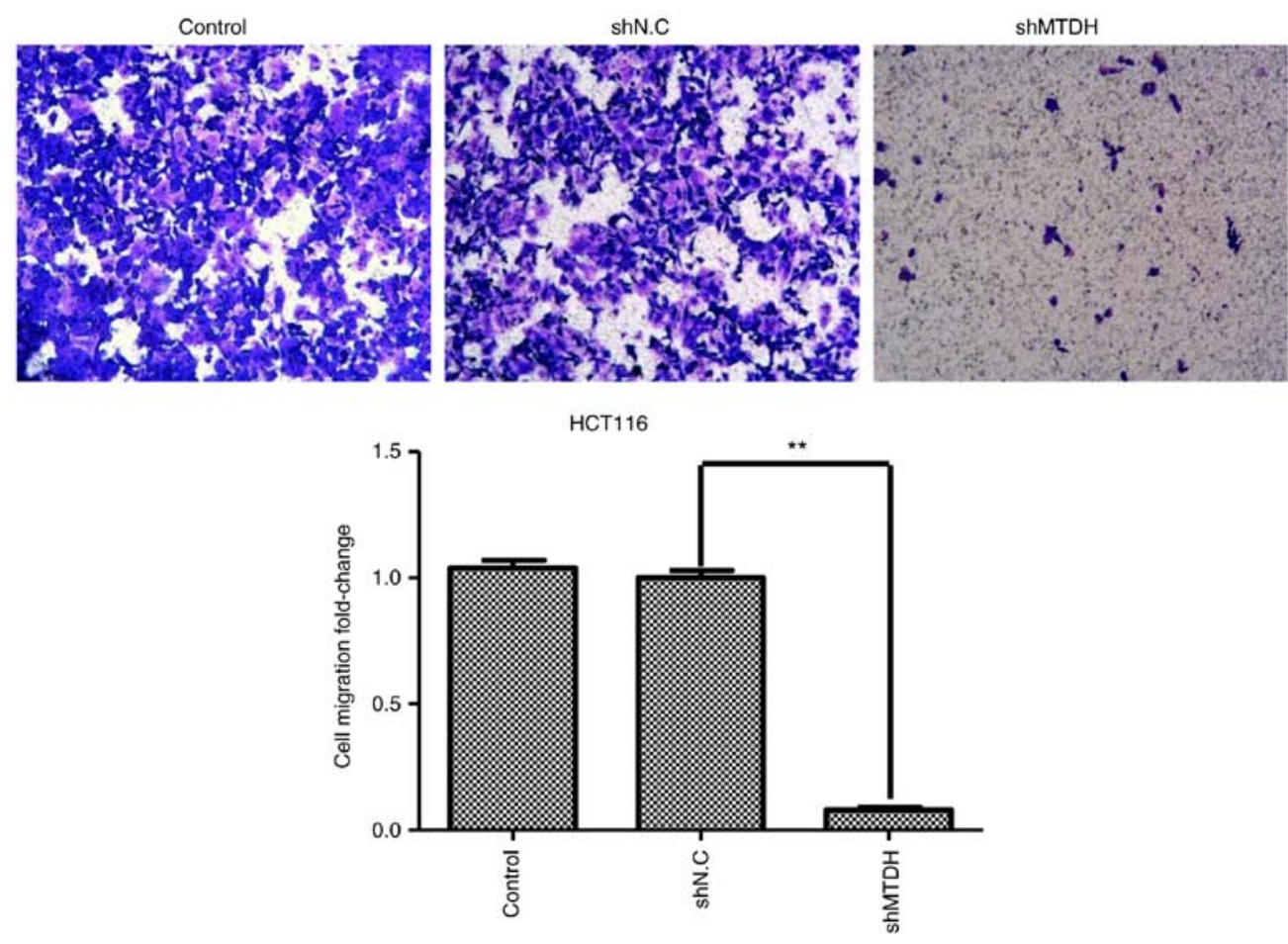

Figure 5. Knockdown of MTDH suppressed the migration of HCT116 cells. A Transwell chamber assay was performed to determine the migration of blank control, and shNC- and shMTDH-transfected HCT116 cells. Cells were allowed to migrate for $48 \mathrm{~h}$ and crystal violet was used to stain the migrating cells that were quantified under a microscope. Representative images of migrating cells are shown. Cell numbers are expressed as the fold-change relative to NC cells in the graph. Data are expressed as the mean \pm standard deviation. ${ }^{* *} \mathrm{P}<0.01 \mathrm{vs}$. shNC and shMTDH groups. Magnification, $\mathrm{x} 100$. MTDH, metadherin; sh, short hairpin RNA; NC, negative control. 

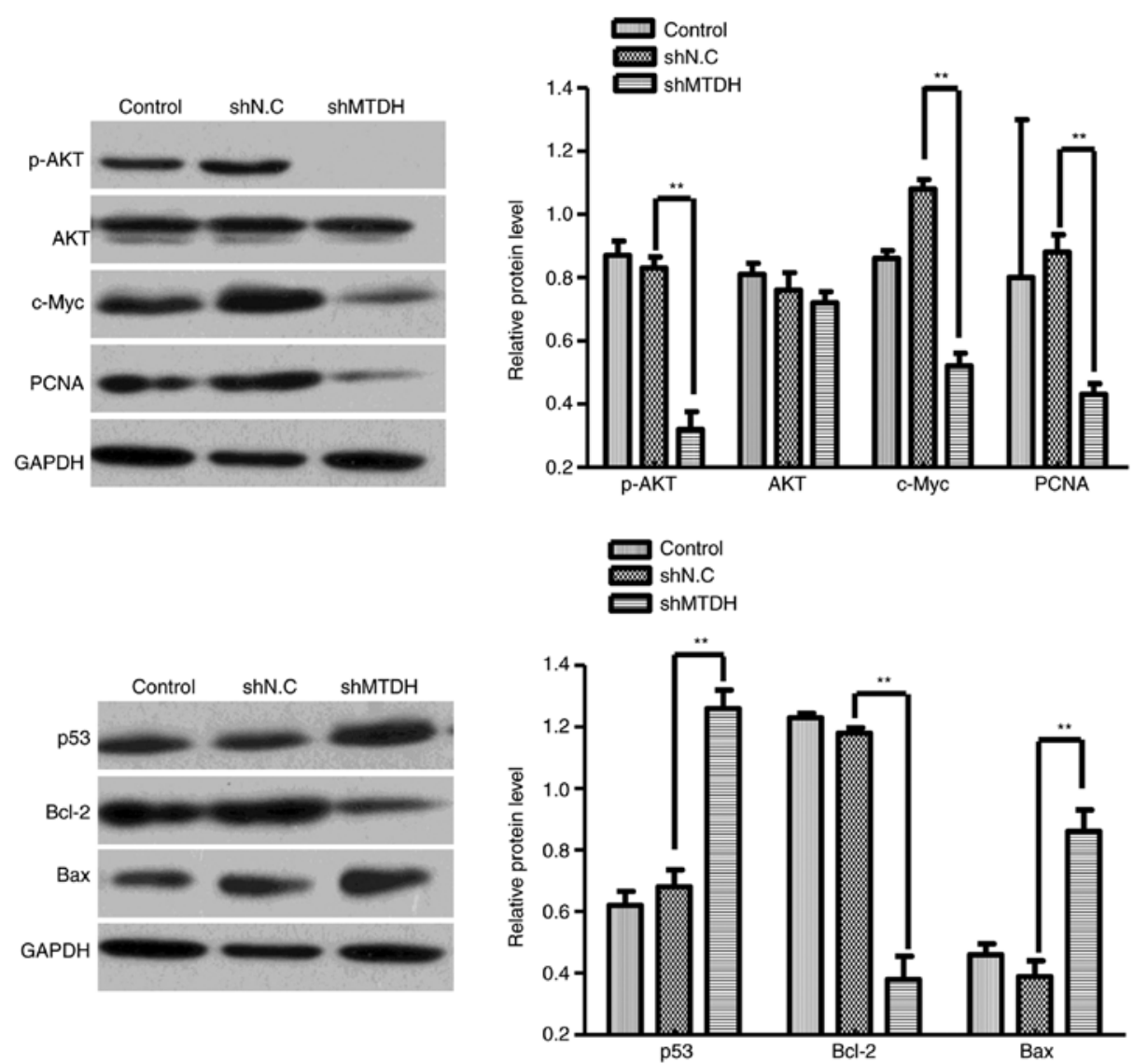

Figure 6. The effects of MTDH-knockdown on the expression of p-Akt, total Akt, c-Myc, PCNA, Bcl-2, p53 and Bax proteins. Western blot analysis was performed to detect the expression of p-Akt, total Akt, c-Myc, PCNA, Bcl-2, p53 and Bax in blank control, and shNC- and shMTDH-transfected HCT116 cells. Data are expressed as the mean \pm standard deviation. ${ }^{* *} \mathrm{P}<0.01$ vs. shNC and shMTDH groups. MTDH, metadherin; p-, phosphorylated; Akt, protein kinase B; PCNA, proliferating cell nuclear antigen; Bcl-2, B-cell lymphoma 2; p53, tumor protein p53; Bax, Bcl-2-associated X protein; NC, negative control.

CRC cell growth and migration, the present study examined the activity of Akt/c-Myc signaling and apoptosis-related protein in shMTDH-transfected HCT116 cells using western blot analysis. As demonstrated in Fig. 6, knockdown of MTDH markedly downregulated the expression of p-Akt, c-Myc and Bcl-2 protein in HCT116 cells, and the expression of p53 and Bax protein was significantly increased compared with the shNC group $(\mathrm{P}<0.05)$. Indicating that MTDH may function through the expression of multiple types of apoptosis-related and signaling channel protein in CRC cells.

Knockdown of MTDH downregulates the expression of $P C N A$. To determine whether the depletion of MTDH can regulate PCNA expression in CRC cells, the protein expression of PCNA in MTDH-deficient HCT116 cells was analyzed. As demonstrated in Fig. 6, the protein expression of PCNA in shMTDH-transfected cells was significantly downregulated compared with that in shNC-transfected cells, suggesting that PCNA may serve as a downstream effector of MTDH to regulate CRC cell growth, apoptosis, cell cycle and migration.

\section{Discussion}

The present study investigated the expression and role of MTDH in CRC cells in vitro and revealed that MTDH was highly expressed in CRC cell lines. In addition, shRNA-mediated knockdown of MTDH significantly inhibited CRC cell proliferative, colony-forming and migratory abilities while inducing cell apoptosis and $\mathrm{S}$ phase cell cycle arrest. Mechanistically, knockdown of MTDH markedly downregulated the protein expression of p-Akt, c-Myc, Bcl-2 and PCNA, while upregulating the protein of $\mathrm{p} 53$ and Bax expression of in HCT116 cells. These results suggested that MTDH-knockdown induces apoptosis of HCT116 cells, and its mechanism may be associated with upregulation of Bax protein expression and downregulation of $\mathrm{Bcl}-2$ protein expression. MTDH is essential for CRC cell growth and migration in vitro possibly through the Akt/c-Myc signaling pathway.

MTDH can be induced by human immunodeficiency virus (HIV)-1 infection or recombinant HIV-1 envelope glycoprotein (gp120) treatment in primary human fetal astrocytes (22). Previous studies have reported that MTDH expression is positively correlated with high risks of cancer in humans $(23,24)$. A recent study demonstrated that the knockdown of MTDH can inhibit ovarian cancer cell growth and invasion while inducing apoptosis and cell cycle arrest at the G0/G1 phase (25). In addition, MTDH was revealed to be highly abundant in HCC cells and tissues, and knockdown of MTDH can suppress HCC cell growth and colony formation in vitro, as well as inhibiting xenografted tumor growth in vivo (26). Furthermore, the present study also demonstrated 
that MTDH depletion significantly inhibits cell proliferation and colony formation while inducing apoptosis and cell cycle arrest at the $\mathrm{S}$ phase in CRC cells. Based on these findings, we hypothesized that MTDH may function as an oncogene and serve a critical role in CRC development and progression.

Metastasis is one of the most critical hallmarks of cancer. The upregulation of MTDH has been demonstrated to promote invasive and metastatic abilities of non-small cell lung cancer cells (23). MTDH overexpression in patients with primary CRC may be considered as a biomarker for lung-specific metastases (27). Consistent with these findings, the present study demonstrated that MTDH depletion can suppress CRC cell migration (Fig. 5), indicating that MTDH is possibly involved in CRC metastasis and may serve as a novel biomarker for CRC metastasis. Targeting MTDH may be a potential therapeutic strategy against CRC.

A previous study has demonstrated that MTDH regulates malignancy development through various cellular signaling cascades, including the MAPK, Ha-ras, NF- $\mathrm{KB}, \mathrm{PI} 3 \mathrm{~K} / \mathrm{Akt}$ and Wnt pathways (28). Akt signaling is important for regulating tumor cell proliferation, invasion, apoptosis, cell cycling and survival. Previous studies have demonstrated that the $\mathrm{PI} 3 \mathrm{~K} / \mathrm{Akt}$ signaling pathway is aberrantly activated in human cancer $(29,30)$. Although the overexpression of Akt frequently occurs during CRC carcinogenesis, this is not the case in CRC with microsatellite instability (31). c-Myc is a protooncogene (32) that regulates cell growth and proliferation through regulating a number of downstream target genes, serving an oncogenic role in multiple types of human cancer (33). The upregulation of c-Myc significantly enhances the invasive and metastatic abilities of cancer cells (34). Another previous study demonstrated that MTDH expression can be induced by Ha-ras via the PI3K-Akt signaling pathway (16). MTDH also affects Akt phosphorylation, which is involved in c-Myc-suppressed apoptosis (35). Therefore, as one of the downstream effectors of c-Myc and Ha-ras signaling, MTDH may serve a key role in tumor development and progression (16). The present study consistently indicated that knockdown of MTDH leads to a markedly reduced expression of c-Myc, p-Akt, Bcl-2 and PCNA. MTDH shRNA also upregulated the protein expression of p53 and Bax in HCT116 cells, suggesting that MTDH silencing inhibits CRC cell proliferation and migration. MTDH-knockdown induces apoptosis possibly by upregulation of Bax protein expression and downregulation of $\mathrm{Bcl}-2$ protein expression, and the Akt and c-Myc signal channel may be involved in the changes in protein expression. Akt activation may inhibit its downstream target glycogen synthase kinase-3, which phosphorylates c-Myc at 58 asinine residue, resulting in the inhibition of c-Myc protein degradation (36,37). Therefore, MTDH-activated Akt signaling may cause an upregulation of the protein level of c-Myc, which further promotes CRC development.

In summary, the results of the present study demonstrated that MTDH expression is commonly expressed in CRC cell lines and shRNA-mediated knockdown of MTDH inhibits HCT116 cell proliferation, colony formation and migration while inducing cell cycle arrest at the $\mathrm{S}$ phase and apoptosis, which is not only associated with the downregulation of p-Akt, c-Myc, Bcl-2 and PCNA expression, but also associated with the upregulation of p53 and Bax protein. Further studies are required to elucidate the mechanisms underlying the regulatory activity of MTDH in CRC. Therefore, targeting MTDH may be a potential therapeutic strategy in CRC treatment. Further in vivo investigation is required to develop MTDH inhibitors for CRC therapy.

\section{Acknowledgements}

Not applicable.

\section{Funding}

The present study was supported by the Foundation of Haikou Science and Technology Bureau, Haikou, Hainan, China (grant no, 2015-035).

\section{Availability of data and materials}

The datasets used and/or analyzed during the current study are available from the corresponding author on reasonable request.

\section{Authors' contributions}

JWL and CZH performed the research, the data acquisition and drafted the manuscript; JHL, JHY, YPL, WFZ, MXZ, YL and $\mathrm{QHC}$ provided assistance for data acquisition, data analysis and statistical analysis; LGL designed the research, edited and reviewed the manuscript. ZSX provided assistance on the data acquisition, data analysis and statistical analysis. All authors have read and approved the content of the manuscript.

\section{Ethics approval and consent to participate}

Not applicable.

\section{Patient consent for publication}

Not applicable.

\section{Competing interests}

All authors declare that they have no competing interests.

\section{References}

1. Theodoratou E, Farrington SM, Tenesa A, McNeill G, Cetnarskyj R, Korakakis E, Din FV, Porteous ME, Dunlop MG and Campbell H: Associations between dietary and lifestyle risk factors and colorectal cancer in the Scottish population. Eur J Cancer Prev 23: 8-17, 2014.

2. Akagi Y, Kinugasa T, Adachi Y and Shirouzu K: Prognostic significance of isolated tumor cells in patients with colorectal cancer in recent 10-year studies. Mol Clin Oncol 1: 582-592, 2013.

3. Guo Y, Xu F, Lu T, Duan Z and Zhang Z: Interleukin-6 signaling pathway in targeted therapy for cancer. Cancer Treat Rev 38: 904-910, 2012.

4. Lièvre A, Blons $\mathrm{H}$ and Laurent-Puig P: Oncogenic mutations as predictive factors in colorectal cancer. Oncogene 29: 3033-3043, 2010.

5. Grady WM and Pritchard CC: Molecular alterations and biomarkers in colorectal cancer. Toxicol Pathol 42: 124-139, 2014.

6. Kang DC, Su ZZ, Sarkar D, Emdad L, Volsky DJ and Fisher PB: Cloning and characterization of HIV-1-inducible astrocyte elevated gene-1, AEG-1. Gene 353: 8-15, 2005. 
7. Hu G, Wei Y and Kang Y: The multifaceted role of MTDH/AEG-1 in cancer progression. Clin Cancer Res 15: 5615-5620, 2009.

8. Li M, Dai Y, Wang L and Li L: Astrocyte elevated gene-1 promotes the proliferation and invasion of breast cancer cells by activating the $W n t / \beta$-catenin signaling pathway. Oncol Lett 13 : 2385-2390, 2017.

9. Yao Y, Gu X, Liu H, Wu G, Yuan D, Yang X and Song Y: Metadherin regulates proliferation and metastasis via actin cytoskeletal remodelling in non-small cell lung cancer. $\mathrm{Br}$ J Cancer 111: 355-364, 2014.

10. Zhu HD, Liao JZ, He XX and Li PY: The emerging role of astrocyte elevated gene in hepatocellular carcinoma (Review). Oncol Rep 34: 539-546, 2015.

11. Jung HI, Ahn T, Bae SH, Chung JC, Kim H, Chin S, Jeong D, Cho HD, Lee MS, Kim HC, et al: Astrocyte elevated gene-1 overexpression in hepatocellular carcinoma: An independent prognostic factor. Ann Surg Treat Res 88: 77-85, 2015.

12. Jian-bo X, Hui W, Yu-long H, Chang-hua Z, Long-juan Z, Shirong C and Wen-hua Z: Astrocyte-elevated gene-1 overexpression is associated with poor prognosis in gastric cancer. Med Oncol 28: 455-462, 2011

13. Tokunaga E, Nakashima Y, Yamashita N, Hisamatsu Y, Okada S, Akiyoshi S, Aishima S, Kitao H, Morita M and Maehara Y: Overexpression of metadherin/MTDH is associated with an aggressive phenotype and a poor prognosis in invasive breast cancer. Breast Cancer 21: 341-349, 2014.

14. Gnosa S, Shen YM, Wang CJ, Zhang H, Stratmann J, Arbman G and Sun XF: Expression of AEG-1 mRNA and protein in colorectal cancer patients and colon cancer cell lines. J Transl Med 10: 109, 2012.

15. Emdad L, Sarkar D, Su ZZ, Randolph A, Boukerche H, Valerie K and Fisher PB: Activation of the nuclear factor kappaB pathway by astrocyte elevated gene-1: Implications for tumor progression and metastasis. Cancer Res 66: 1509-1516, 2006.

16. Lee SG, Su ZZ, Emdad L, Sarkar D and Fisher PB: Astrocyte elevated gene-1 (AEG-1) is a target gene of oncogenic Ha-ras requiring phosphatidylinositol 3-kinase and c-Myc. Proc Nat Acad Sci USA 103: 17390-17395, 2006.

17. Wei J, Li Z, Chen W, Ma C, Zhan F, Wu W and Peng Y: AEG-1 participates in TGF-betal-induced EMT through p38 MAPK activation. Cell Biol Int 37: 1016-1021, 2013.

18. Li PP, Feng LL, Chen N, Ge XL, Lv X, Lu K, Ding M, Yuan D and Wang X: Metadherin contributes to the pathogenesis of chronic lymphocytic leukemia partially through Wnt/ $\beta$-catenin pathway. Med Oncol 32: 21, 2015.

19. Song H, Li C, Li R and Geng J: Prognostic significance of AEG-1 expression in colorectal carcinoma. Int J Colorectal Dis 25 1201-1209, 2010.

20. Livak KJ and Schmittgen TD: Analysis of relative gene expression data using real-time quantitative PCR and the $2^{-\Delta \Delta C_{\mathrm{T}}}$ method. Methods 25: 402-408, 2001.

21. Wang F, Wang H, Sun X and Li M: Apoptosis-induction is a novel therapeutic strategy for gastrointestinal and liver cancers. Curr Gene Ther 15: 193-200, 2015.

22. Su ZZ, Kang DC, Chen Y, Pekarskaya O, Chao W, Volsky DJ and Fisher PB: Identification and cloning of human astrocyte genes displaying elevated expression after infection with HIV-1 or exposure to HIV-1 envelope glycoprotein by rapid subtraction hybridization, RaSH. Oncogene 21: 3592-3602, 2002.
23. Sun S, Ke Z, Wang F, Li S, Chen W, Han A, Wang Z, Shi H, Wang LT and Chen X: Overexpression of astrocyte-elevated gene-1 is closely correlated with poor prognosis in human non-small cell lung cancer and mediates its metastasis through up-regulation of matrix metalloproteinase- 9 expression. Hum Pathol 43: 1051-1060, 2012.

24. Yu JQ, Zhou Q, Zhu H, Zheng FY and Chen ZW: Overexpression of astrocyte elevated gene-1 (AEG-1) in cervical cancer and its correlation with angiogenesis. Asian Pac J Cancer Prev 16: 2277-2281, 2015

25. Wang J, Chen X and Tong M: Knockdown of astrocyte elevated gene-1 inhibited cell growth and induced apoptosis and suppressed invasion in ovarian cancer cells. Gene 616: 8-15, 2017.

26. Li WF, Ou Q, Dai H and Liu CA: Lentiviral-mediated short hairpin RNA knockdown of MTDH inhibits cell growth and induces apoptosis by regulating the PTEN/AKT pathway in hepatocellular carcinoma. Int J Mol Sci 6: 19419-19432, 2015.

27. Casimiro S, Fernandes A, Oliveira AG, Franco M, Pires R, Peres M, Matias M, Tato-Costa J, Guerra N, Ramos M, et al: Metadherin expression and lung relapse in patients with colorectal carcinoma. Clin Exp Metastasis 31: 689-696, 2014.

28. Shi $X$ and Wang X: The role of MTDH/AEG-1 in the progression of cancer. Int J Clin Exp Med 8: 4795-4807, 2015.

29. Millis SZ, Ikeda S, Reddy S, Gatalica Z and Kurzrock R: Landscape of phosphatedy- linositol-3-kinase pathway alterations across 19784 diverse solid tumors. JAMA Oncol 2: 1565-1573, 2016.

30. Brown JS and Banerji U: Maximising the potential of AKT inhibitors as anti-cancer treatments. Pharmacol Ther 172: 101-105, 2017.

31. Roy HK, Olusola BF, Clemens DL, Karolski WJ, Ratashak A, Lynch HT and Smyrk TC: AKT proto-oncogene over-expression is an early event during sporadic colon carcinogenesis. Carcinogenesis 23: 201-205, 2002.

32. Nesbit CE, Tersak JM and Prochownik EV: MYC oncogenes and human neoplastic disease. Oncogene 18: 3004-3016, 1999.

33. Kalkat M, De Melo J, Hickman KA, Lourenco C, Redel C, Resetca D, Tamachi A, Tu WB and Penn LZ: MYC deregulation in primary human cancers. Genes 8: E151, 2017.

34. Liu Z, He Q, Ding X, Zhao T, Zhao L and Wang A: SOD2 is a $\mathrm{C}$-myc target gene that promotes the migration and invasion of tongue squamous cell carcinoma involving cancer stem-like cells. Int J Biochem Cell Biol 60: 139-146, 2015.

35. Lee SG, Su ZZ, Emdad L, Sarkar D, Franke TF and Fisher PB: Astrocyte elevated gene-1 activates cell survival pathways through PI3K-Akt signaling. Oncogene 27: 1114-1121, 2008.

36. Gregory MA, Qi Y and Hann SR: Phosphorylation by glycogen synthase kinase-3 controls c-Myc proteolysis and subnuclear localization. J Biol Chem 278: 51606-51612, 2003.

37. Lepique AP, Moraes MS, Rocha KM, Eichler CB, Hajj GN, Schwindt TT and Armelin HA: c-Myc protein is stabilized by fibroblast growth factor 2 and destabilized by ACTH to control cell cycle in mouse Y1 adrenocortical cells. J Mol Endocrinol 33: 623-638, 2004 